\title{
A New Energy-Efficient On-Demand Routing Protocol for Green Wireless Mesh Networks
}

\author{
Yinpeng Yu, Yuhuai Peng, Lei Guo, Meng Song \\ College of Information Science and Engineering, Northeastern University, Shenyang, 110819, China \\ E-mail: haveball@gmail.com
}

\begin{abstract}
In order to solve the green energy saving problem with the wireless routing technology, a new energy-efficient survivable routing protocol, called Energy-efficient Survivable AODV (ES-AODV) for unicast demand in green Wireless Mesh Networks (WMNs), is proposed in this paper. The routing protocol uses the hop penalty strategy and flooding delaying strategy to improve the performances of traditional AODV routing protocol. This paper uses QualNet to simulate and verify the performances of proposed ES-AODV. The simulation results show that, compared with the traditional AODV, the energy utilization of ES-AODV is more efficient than AODV by $13 \%$. At the same time, ES-AODV is more load-balancing to reduce the number of energy depletion nodes and postpone the appearance of the first energy depletion node, and thus it effectively improves the network survivability.
\end{abstract}

Keywords-green Wireless Mesh Networks; energy-efficient; survivable; routing protocol

\section{INTRODUCTION}

With the rapid development of wireless communication and wireless network technology, Wireless Mesh Network (WMN) has emerged. As a new wireless access network, the kernel idea of WMN is to allow each node of the network to send and receive signals, so that, the problems of traditional wireless networks, such as poor robustness and low scalability, can be well solved.

WMN is now being used to deploy Wi-Fi coverage in a wide variety of outdoor applications [1]. In WMN, conventional mesh nodes must be operated using continuous electrical power connections. This requirement may often be very expensive, especially when the network includes expansive outdoor wireless coverage areas. In order to replace this kind of fixed energy supply way, solar energy, wind energy and other renewable energy can be used to provide support for the node energy [2]. Thus, this paper focuses on the green WMNs which are based on solar energy and wind mixed for node power supply.

As one of the key technologies in WMN, wireless routing plays an important role in the performance optimization of WMN. Since there are no official routing protocol standards for green WMNs so far, it is necessary to design specialized and efficient routing protocols for green WMNs.

The energy-efficient routing protocol in green WMNs can better combine the green energy saving and energy balance with the wireless routing technology [3]. Thus, it can provide technical support for the area which is rich in solar and wind energy to develop a new kind of green WMNs.

The rest of this paper is organized as follows: Section 2 gives the details of proposed ES-AODV protocol. Section 3 shows the simulation results and analysis of these protocols. Section 4 concludes this paper.

\section{ES-AODV PROTOCOL}

In order to use the energy of each node reasonably and balance the energy consumption to improve the energy efficiency in green WMNs, it is necessary to know the remaining energy of each current node. If using table-driven routing protocols to find routes, it needs to broadcast routing update information frequently to let other nodes know the remaining energy of the current node, which will give the network great burden. However on-demand routing protocols find nodes' information in the routing discovery process, and they initiate the routing discovery on-demand to establish the route dynamically when there are no efficient route from the source node to the destination node [4]. Therefore, this paper is based on the widely used AODV protocol of on-demand routing protocols, to propose an improved routing protocol.

\section{A. Hop penalty strategy}

Traditional AODV protocol uses the minimum hops as routing metric, in order to reduce the routing establishment time and decrease the control overhead [5]. But this metric may cause multiple routes to traverse the same node, which will increase the load of this node. This situation may lead to local congestion of network, and some idle paths do not be efficiently used, which wastes the energy of nodes in these paths [6]. At the same time, the nodes passed by multiple routes may be out of energy rapid, and then the network will be divided into pieces and cannot be interconnected, which affects the network survivability. Therefore, it is necessary to take into account the nodes' remaining energy in the routing discovery and routing selection.

\section{1) Definition of ES-AODV metric}

This paper defines a new routing metric, which converts the remaining energy of the nodes into hop penalty factor. Along with forwarding RREQ and RREP packets, the punished hops (i.e., routing cost) are accumulated constantly. When receiving RREP, the source nose has the same process to choose the route of the minimum punished hops as the transmission path. Therefore, the hops and remaining energy will be combined in the new routing metric. 
We suppose the $j^{\text {th }}$ route as route $e_{j}=\left(n_{o}, n_{1}, n_{2}, \ldots, n_{k}, n_{d}\right)$, of which $n_{o}$ is source node, $n_{d}$ is destination node and $n_{k}$ is the $k^{\text {th }}$ intermediate node. We set $e_{i}(t)$ as the remaining energy of node $n_{i}$ at the time of $t, e_{\text {total }}$ is the total energy of each node and $W$ is the energy penalty weight.

The energy penalty function for node $n_{i}$ can be written as:

$$
C_{i}(t)=1+\left[\left(1-e_{i}(t) / e_{\text {total }}\right) \times W\right]
$$

Where $C_{i}(t)$ means the routing cost of node $n_{i}$, which represents the remaining energy of node $n_{i}$. From the function, we can see that the value of $C_{i}(t)$ becomes high with the decrease of remaining energy of node $n_{i}$.

Energy penalty weight $W$ is the specific weight between hops and remaining energy of nodes. When $W$ increases, the remaining energy of nodes will have a high specific weight in routing metric and the route has good energy efficiency performance. If $W$ is set as 0 , the hops don't have punishment and the metric is the same as AODV protocol. In the simulation, the energy penalty weight $W$ is set as 20 .

The routing cost function of route ${ }_{j}$ can be formulated as:

$$
C_{j}=\sum_{i=o}^{d} C_{i}(t)
$$

Obviously, this function is the sum of all routing cost of nodes in route . $_{\text {. }}$.

Then, the optimal route is described as:

$$
\text { route }_{\text {optimal }}=\text { route }_{j^{*}}
$$

where $C_{j^{*}}=\min _{j}\left\{C_{j} \mid j=1,2, \ldots, n\right\}$. It is clearly that route $j^{*}$ with the minimum cost is selected as the optimal route.

2) Energy constrained routing diameter

In the standard AODV protocol, a constant NET_DIAMETER is defined to restrict the forward of RREQ packets in the network [7]. The TTL in IP header is set as NET_DIAMETER to limit the searching range of RREQ packets. In other words, the RREQ can only be forwarded for NET_DIAMETER times and this value is set as 35 in general [8]. To imitate this idea, this paper defines an energy constrained routing diameter ES_DIAMETER, which is used to restrict the forward of RREQ and RREP packets. When $\sum_{i=0}^{j} f_{i}(t)>$ ES_DIAMETER, the accumulation of punished hops is beyond ES_DIAMETER. Therefore, the RREQ or RREP packets are directly discarded.

Setting a suitable value of ES_DIAMETER can guarantee good routing information transmitted in the network to achieve routing discovery process, which can reduce the control overhead. But the value of ES DIAMETER should not be set too small. Because in case that each node's remaining energy in the network is generally little, the source node will cannot find a route to the destination node. In the simulation of the paper, the value of ES_DIAMETER is set as 35.

\section{B. Flooding delaying strategy}

In AODV protocol, when receiving RREQ packets the intermediate nodes will randomly delay a period of time before forwarding them, in order to avoid broadcast storm [9]. This time equals to the product of 0.01 second and a value obeying random distribution of $[0,1]$ [10]. The protocol can indirectly control the arrival time of RREP packets and further affect the earliest arriving principle of AODV routing metric to control the flooding delay.

\section{1) Problem about hop penalty strategy}

The hop penalty strategy can significantly balance the energy consumption. If most of nodes in a route have much remaining energy and only a few nodes have little remaining energy, in this way the routing cost of the entire route is not very high and this route may be chosen. But when this route is used to transmit data, the nodes with little remaining energy will use out all energy, which threatens the processing of the traffic and the network survivability.

In order to avoid the above problem and further optimize the protocol's performance on balancing the energy consumption, the paper proposes the flooding delaying strategy. This strategy will delay the nodes (i.e., high-risk nodes) of which the remaining energy is less than a certain threshold for a period of time to broadcast messages. This strategy is a supplement to the hop penalty strategy.

\section{2) Definition of flooding delaying strategy}

We define $b_{\text {threshold }}$ as the threshold of node's remaining energy and $T_{\text {random }}$ as a decimal value obeying random distribution of $[0,1] . T_{c}$ is the delay constant. In this paper, $b_{\text {threshold }}$ is set as $20 \%$ and $T_{c}$ is set as 0.05 second.

The delay time is defined as:

$$
T=\left\{\begin{array}{l}
T_{\text {random }} \times 0.01 s,\left(b_{i}(t) / b_{i} \max \geq b_{\text {threshold }}\right) \\
\left(1-b_{i}(t) / b_{i} \max \right) \times T_{c},\left(b_{i}(t) / b_{i} \max <b_{\text {threshold }}\right)
\end{array}\right.
$$

When node forwards RREQ packets, it must read the value of remaining energy firstly. By comparing this value with the threshold, the protocol can know whether this node is high-risk and determine when to flood RREQ packets after a period of time.

\section{SIMULATION RESULTS AND ANALYSIS}

In this paper, the simulation software is QualNet, which is a state-of-the-art simulator for large, heterogeneous networks and the distributed applications that execute on those networks.

In order to study the overall performances of the ESAODV protocol, this paper has done comprehensive simulation on the platform of QualNet Developer 5.0.2. In the simulation, the network consists of 50 mesh router nodes, which are randomly distributed in a square region of $4,000 \times 4,000$ square meters, and the simulation time is 500 seconds. The traffic mode is CBR and the size of data packet for sending is 512 bytes. Since this paper focuses on the energy-efficient of WMN, the simulation is unlike the general routing protocols' traffic setting. The paper assumes that the WMN works in a wireless city environment of high 
load. The application of CBR is randomly started and continues for a fixed duration, which makes it close to reality and focuses on the analysis of the energy consumption.

The performance indexes tested are as follows:

Average remaining energy of nodes: it is the average value of the remaining energy of all the nodes in the network, which is defined by the following formula:

$$
A R E=\frac{1}{n} \sum_{j=1}^{n} E_{j}
$$

Average remaining energy of nodes is used to measure the situation of nodes' energy. In the formula, $E_{j}$ represents the remaining energy of node $j$ and $n$ represents the number of nodes in the route.

Unbalanced degree of remaining energy of nodes: it is the standard deviation of the remaining energy in all the nodes, which is defined by the following formula:

$$
U D R E=\sqrt{\frac{1}{n} \sum_{j=1}^{n} E_{j}^{2}-\left(\frac{1}{n} \sum_{j=1}^{n} E_{j}\right)^{2}}
$$

Unbalanced degree of remaining energy of nodes measures the difference of the nodes' remaining energy. The energy consumption of nodes is not balanced and nodes' energy is easily depleted when the value of unbalanced degree of remaining energy of nodes becomes large.

The number of nodes with energy exhaustion: it means the number of energy depleted nodes in the network, which is derived from the statistics of simulation software. Along with simulation time, energy depleted nodes may appear. The number of energy depleted nodes and their appeared time reflect the performance of the routing protocol to improve the network survivability and energy balancing.

Variance of idle ratio of nodes: it is refers to the variance of the ratio of the network nodes in idle state, which is defined by the following formula:

$$
V I R=\frac{1}{n} \sum_{j=1}^{n} I_{j}^{2}-\left(\frac{1}{n} \sum_{j=1}^{n} I_{j}\right)^{2}
$$

Variance of idle ratio of nodes measures the differences in the network load distribution. In the formula, $I_{j}$ represents the ratio of node $j$ in idle state. The low value of variance means that the protocol assigns load averagely.

\section{A. Performance of average remaining energy of nodes}

Table 1 is the energy consumption comparison between AODV protocol and ES-AODV protocol. In this paper, the nodes are assumed to use battery for energy, thus the unit of energy is set as milli-Ampere-hour (mAh) for battery capacity. The ratio of energy consumption in the table is calculated from the energy consumption of single CBR of AODV divided by that of ES-AODV. It shows that the energy utilization of ES-AODV is more efficient than AODV by $13 \%$ when dealing with single traffic of CBR.
TABLE I. COMPARISON OF THE ENERGY CONSUMPTION BETWEEN AODV AND ES-AODV

\begin{tabular}{|c|c|c|}
\hline \multirow{2}{*}{ Comparison } & \multicolumn{2}{|c|}{ Protocol } \\
\cline { 2 - 3 } & AODV & ES-AODV \\
\hline Total energy (mAh) & 300 & 300 \\
\hline Average remaining energy (mAh) & 70.42 & 96.97 \\
\hline Average energy consumption (mAh) & 229.58 & 203.03 \\
\hline Energy consumption of single CBR (mAh) & 9.18 & 8.12 \\
\hline Ratio of energy consumption & \multicolumn{2}{|c|}{1.13} \\
\hline
\end{tabular}

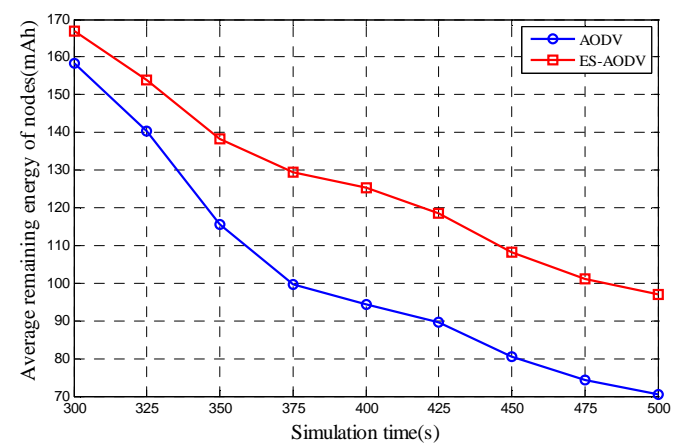

Figure 1. Comparison of average remaining energy of nodes

In Fig.1, it shows that the average remaining energy of nodes becomes low with the increase of simulation time. The average remaining energy of nodes of ES-AODV protocol is higher than that of AODV protocol, which means that ESADOV has obvious effect in the energy saving.

\section{B. Performance of unbalanced degree of remaining energy} of nodes

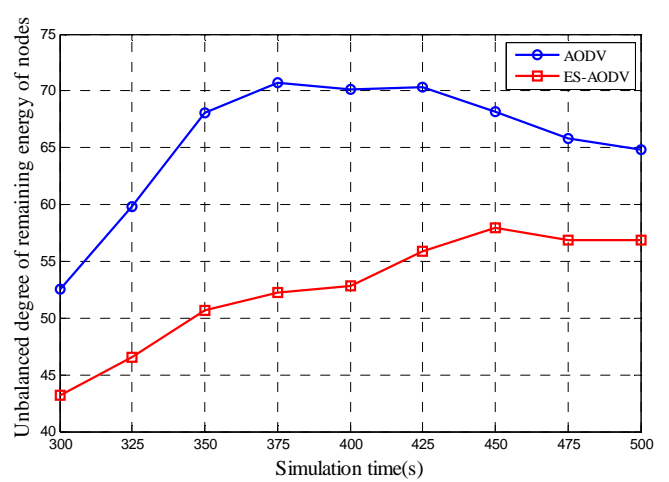

Figure 2. Comparison of unbalanced degree of remaining energy of nodes

It is shown that in Fig.2, the curve of ES-AODV protocol is lower than that of AODV protocol, which means that ESAODV has better performance of energy balancing. At the beginning of simulation, the remaining energy of nodes is the same and unbalanced degree of remaining energy is zero. Along with the simulation time, different nodes have different energy consumption, and unbalanced degree of remaining energy of nodes increases. Since the traffic of CBR is randomly started from 0 to 440 seconds and lasts 60 seconds, after 450 seconds, the traffic and the energy consumption of nodes decreases, and the unbalanced degree tends towards stability. 
C. Performance of the number of nodes with energy exhaustion

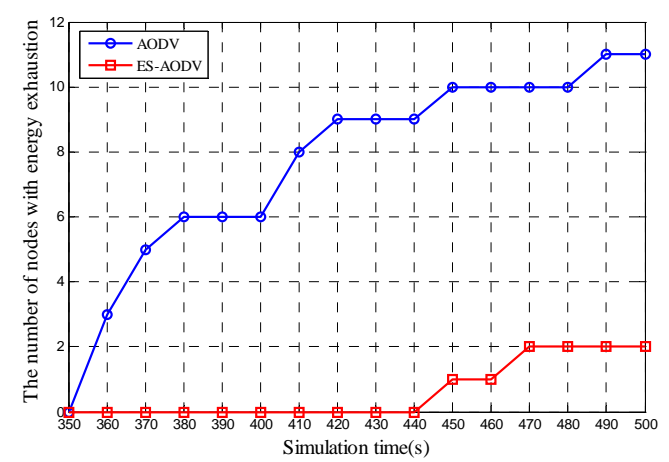

Figure 3. Comparison of the number of nodes with energy exhaustion

In Fig.3, we can see that the number of nodes with energy exhaustion of ES-AODV is significant less than that of AODV. At the same time, the occurrence time of the first node with energy exhaustion of ES-AODV is slower than that of AODV. This is because ES-AODV decreases the number of failed nodes with energy exhaustion by balancing energy consumption to reduce the possibility of link breakage, from failure repaired strategy to failure avoid strategy, so as to improve network survivability. From this performance, it shows that ES-AODV protocol can significantly improve the survivability of WMN with limited energy.

\section{Performance of variance of idle ratio of nodes}

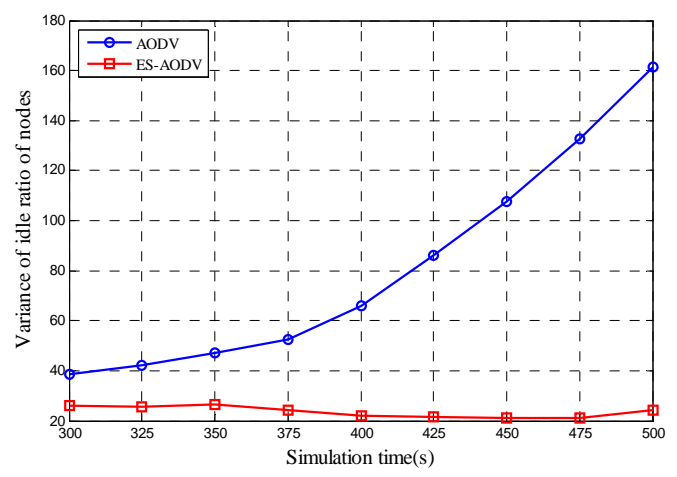

Figure 4. Comparison of variance of idle ratio of nodes

AODV protocol does not consider the energy of the node and simply adopts the minimum hops as a routing metric, which may lead some nodes to be used excessively, energy to be depleted and traffic to be congested. It is shown in Fig.4 that the variance of idle ratio of nodes of AODV protocol has been growing, which means that the unbalanced degree of network load distribution is high. However the variance of idle ratio of nodes of ES-AODV protocol has been relatively stable, which is because ES-ADOV considers the nodes' remaining energy in the routing metric and it can use the hop penalty strategy and flooding delaying strategy to select a route with much remaining energy. Therefore, ESAODV protocol can balance energy consumption and the network load.

\section{CONCLUSION}

This paper proposes an energy-efficient based survivable routing protocol, called ES-AODV. It uses the hop penalty strategy and flooding delaying strategy to improve the traditional AODV routing protocol. The hop penalty strategy is able to achieve the trade-off between the small routing hops and the large node's remaining energy. The flooding delaying strategy can avoid the large delay during routing discovery process. This paper uses QualNet to simulate and verify the proposed ES-AODV. The simulation results show that, the energy utilization of ES-AODV is more efficient than AODV. At the same time, ES-AODV is more loadbalancing to reduce the number of energy depletion nodes and postpone the appearance of the first energy depletion node.

\section{ACKNOWLEDGMENT}

This work was supported in part by the National Natural Science Foundation of China (61172051, 61071124), the Fok Ying Tung Education Foundation (121065), the Program for New Century Excellent Talents in University (11-0075), the Fundamental Research Funds for the Central Universities (N110204001, N110604008), and the Specialized Research Fund for the Doctoral Program of Higher Education (20110042110023, 20110042120035).

\section{REFERENCES}

[1] Y. Zhang, J. Luo, and H. Hu, Wireless mesh networking: architectures, protocols and standards. Beijing, Electronics Industry Publisher, 2008.

[2] L. X. Cai, "Adaptive resource management in green mesh networks with sustainable energy supply," Proc. IEEE GLOBECOM, Dec. 2011.

[3] Z. Niu, "Cell zooming for cost-efficient green cellular networks," IEEE Communications Magazine, vol. 48, pp. 74-79, 2010.

[4] J. Yi, and A. Adnane, "Multipath optimized link state routing for mobile ad hoc networks,” Ad Hoc Networks, vol. 9, pp. 28-47, 2011.

[5] F. D. Rango, J. C. Cano, and M. Fotino, “OLSR vs DSR: a comparative analysis of proactive and reactive mechanisms from an energetic point of view in wireless Ad Hoc networks," Computer Communications, vol. 31, pp. 3843-3854, 2008.

[6] G. Xu, and J. Liu, "Improvement of AODV routing protocol based on wireless mesh networks,” Proc. CMCE, Aug. 2010, pp. 372-375.

[7] B. Xue, P. Ren, and S. Yan, "Link optimization ad-hoc on-demand multipath distance vector routing for mobile ad-hoc networks," Proc. WICOM, Sept. 2009, pp. 2894-2899.

[8] Y. Liu, and L. Han, "The research on an AODV-BRL to increase reliability and reduce routing overhead in MANET,” Proc. ICCASM, oct. 2010, pp. 526-530.

[9] R. Hou, K. Lui, and F. Baker, "Hop-by-hop routing in wireless mesh networks with bandwidth guarantees," IEEE Transactions on Mobile Computing, vol. 11, pp. 264-277, 2012.

[10] S. Kumar, and J. Sengupta, "AODV and OLSR routing protocols for wireless ad-hoc and mesh networks,” Proc. ICCCT, Sept. 2010, pp. 402-407. 